\title{
Existence, Character, and Origin of Surface-Related Bands in the High Temperature Iron Pnictide Superconductor $\mathrm{BaFe}_{2-x} \mathrm{Co}_{x} \mathrm{As}_{2}$
}

\author{
Erik van Heumen, ${ }^{1, *}$ Johannes Vuorinen, ${ }^{2}$ Klaus Koepernik, ${ }^{3}$ Freek Massee,,${ }^{1}$ Yingkai Huang, ${ }^{1}$ Ming Shi, ${ }^{4}$ Jesse Klei, ${ }^{1}$ \\ Jeroen Goedkoop, ${ }^{1}$ Matti Lindroos, ${ }^{2}$ Jeroen van den Brink, ${ }^{3}$ and Mark S. Golden ${ }^{1}$ \\ ${ }^{1}$ van der Waals-Zeeman institute, University of Amsterdam, 1018 XL Amsterdam, the Netherlands \\ ${ }^{2}$ Department of Physics, Tampere University of Technology, PO Box 692, FIN-33101 Tampere, Finland \\ ${ }^{3}$ Institute for Theoretical Solid State Physics, IFW Dresden, D-01171 Dresden, Germany \\ ${ }^{4}$ Paul Scherrer Institut, Swiss Light Source, 5232 Villigen, Switzerland
}

(Received 17 September 2010; published 13 January 2011)

Low energy electron diffraction (LEED) experiments, LEED simulations, and finite slab density functional calculations are combined to study the cleavage surface of Co doped $\mathrm{BaFe}_{2-x} \mathrm{Co}_{x} \mathrm{As}_{2}$ $(x=0.1,0.17)$. We demonstrate that the energy dependence of the LEED data can only be understood from a terminating 1/2 Ba layer accompanied by distortions of the underlying As-Fe 2 -As block. As a result, surface-related $\mathrm{Fe} 3 d$ states are present in the electronic structure, which we identify in angle resolved photoemission spectroscopy (ARPES) experiments. The close proximity of the surface-related states to the bulk bands inevitably leads to broadening of the ARPES signals, which excludes the use of the $\mathrm{BaFe}_{2-x} \mathrm{Co}_{x} \mathrm{As}_{2}$ system for accurate determination of self-energies using ARPES.

The recently discovered iron pnictide superconductors [1] have quickly turned into one of the most widely studied systems in condensed matter research, using all the modern tools that have been perfected through years of research on another class of high temperature superconductors: the cuprates. However, two of the most important and widely used tools to determine the electronic structure, scanning tunneling microscopy (STM) and angle resolved photoemission spectroscopy (ARPES) are surface sensitive techniques. It is therefore crucial to assess the impact of the surface on the electronic structure.

Several different structures containing As- $\mathrm{Fe}_{2}$-As blocks are known: LnOFeAs, $M \mathrm{FeAs}$, and $M \mathrm{Fe}_{2} \mathrm{As}_{2}$, commonly referred to as the "1111," "111," and "122" families. Subdividing the unit cell into As- $\mathrm{Fe}_{2}$-As blocks and spacer layers results in an alternating stack of positively and negatively charged layers. Because of the alternating charges of these layers, the presence of a surface can give rise to a diverging electric potential in the solid $[2,3]$. Such polar surfaces are energetically unfavorable, and usually a structural or electronic reconstruction takes place to prevent their occurrence. LaOFeAs is such an example: cleavage takes place between the $\mathrm{LaO}$ and As- $\mathrm{Fe}_{2}$-As blocks, resulting in either a highly reconstructed La or As termination [4], which supports surface-related states that have been observed in ARPES [5]. The surface of the 111 system, on the other hand, barely influences the electronic structure [6].

This leaves the $M \mathrm{Fe}_{2} \mathrm{As}_{2}$ pnictide superconductors which are by far the most widely studied using surface sensitive probes. In this case, STM experiments show a diversity of topographies resulting from the cleaving process [7-12], which have been interpreted as either consisting of As or Ba layers. Recent first principle calculations for the parent compounds show a reconstructed, $\mathrm{Ba}$ terminated surface to be energetically favorable, leaving open the possibility for a metastable As termination upon low- $T$ cleavage [13]. There is a paucity of wellfounded experimental data on the exact surface structure of the superconducting 122 compounds, in particular, when cleaved at cryogenic temperatures as is usual for ARPES and scanning tunneling spectroscopy (STS) studies. Consequently, the impact of the real surface structure on the electronic states responsible for superconductivity remains an open and important issue.

In this Letter we combine IV-LEED (low energy electron diffraction) experiments and simulations with densityfunctional theory (DFT) slab calculations to investigate the cleavage surface of superconducting, Co-doped Ba122. The data show that the half Ba layer termination has an impact on the underlying $\mathrm{As}-\mathrm{Fe}_{2}$-As block, leading to significant departures from the bulk structure. This gives rise to surface-related $\mathrm{Fe} 3 d$ bands, which, in turn, can be found in our ARPES data. These surface states impede accurate quantitative analysis of (for example, the widths of) ARPES features in the Ba122 systems.

We start with the LEED experiments. Samples were top-post cleaved in situ at pressures better than $5 \times 10^{-11}$ mbar at temperatures under $20 \mathrm{~K}$-following the standard recipes used in ARPES and/or STS studies. For details on samples and experimental procedures, see supplementary materials [14]. The measured LEED patterns for $x=0.1$ and 0.17 samples [Figs. 1(a) and 1(b)] resemble a $2 \times 2$ pattern, which in fact arises from a combination of ordered surface structures as indicated in Fig. 1(c). In order to circumvent the polar surface (type III 

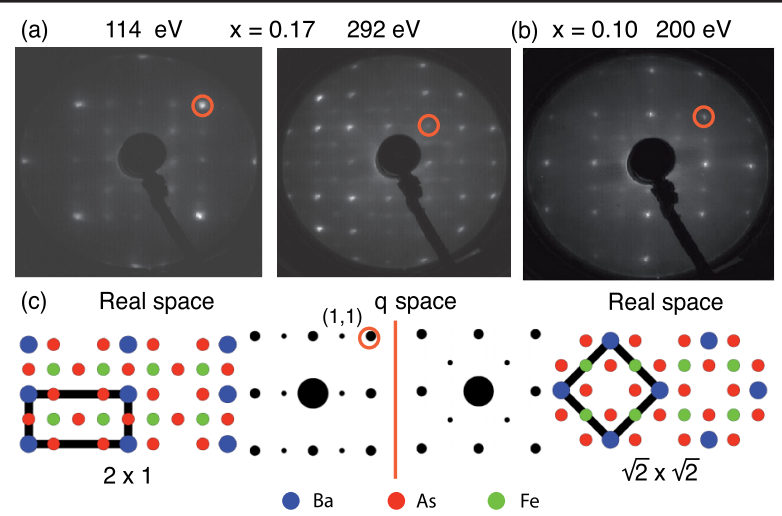

FIG. 1 (color online). (a) Typical LEED images for $E_{0}=114$ and $292 \mathrm{eV}$ for $\mathrm{BaFe}_{2-x} \mathrm{Co}_{x} \mathrm{As}_{2}$ with $x=0.17$. (b) $E_{0}=200 \mathrm{eV}$ for $x=0.1$. In each image, representative $(1,1)$ spots are highlighted with red circles. All images taken at $17 \mathrm{~K}$. (c) Real-space and $q$-space structures of the $2 \times 1$ and $\sqrt{2} \times \sqrt{2}$ structures (green, Ba; purple, As; orange, Fe).

in the classification of Ref. [2]), half of the Ba layer goes with each half of the cleaved crystal. This now places a terminating $1 / 2 \mathrm{Ba}$ layer with charge +1 on top of the underlying As- $\mathrm{Fe}_{2}$-As block which has overall charge -2 . We obtain a zero net dipole moment if we think of the $\mathrm{Ba}$ layer below the uppermost $\mathrm{As}-\mathrm{Fe}_{2}$-As block as half belonging to this upper block and half belonging to the next As- $\mathrm{Fe}_{2}$-As block below. Continuing in this fashion represents the only energetically favorable situation of any likelihood [15]. Ordering in the partial Ba termination layer gives rise to the additional, fractional spots in the LEED images as indicated in Fig. 1(c). The patterns observed are identified as $2 \times 1,1 \times 2$, and $\sqrt{2} \times \sqrt{2}$ ordered partial Ba termination layers. The integer $1 \times 1$ pattern contains higher order reflections from all types of superstructure. The reconstructions are not limited to the tetragonal phase only: the LEED pattern of the underdoped, orthorhombic phase is identical, as shown in Fig. 1(b).

Even though the arguments presented above on the basis of energetics are already persuasive, we now prove the $1 / 2$ Ba termination using IV-LEED data, in which we solve for the real surface structure of the outermost four atomic layers. Figure 2 presents the results from an $x=0.17$ sample, together with the relevant simulations, carried out using the Barbieri-van Hove SATLEED package [16] (see supplementary material [14] for details). Pendry $R$ factors [17] are used to measure the level of agreement between experiment and simulation. Simulations assuming a bulk crystal terminated by a $1 / 2 \mathrm{Ba}$ layer with either $\sqrt{2} \times \sqrt{2}$ or $2 \times 1$ superstructure resulted in overall Pendry $R$ factors, $R_{P} \approx 0.31$ and $R_{P} \approx 0.46$, respectively. As a satisfactory solution should have $R_{p}<0.3$, the ordered superstructures of the half $\mathrm{Ba}$ layer alone are not sufficient. We therefore allowed the atomic positions of the deeper lying layers to relax. Given the measured energy ranges (in total $1208 \mathrm{eV}$ for the $\sqrt{2} \times \sqrt{2}$ and $1782 \mathrm{eV}$ for the $2 \times 1$ superstructure), optimizing the parameters of the first four

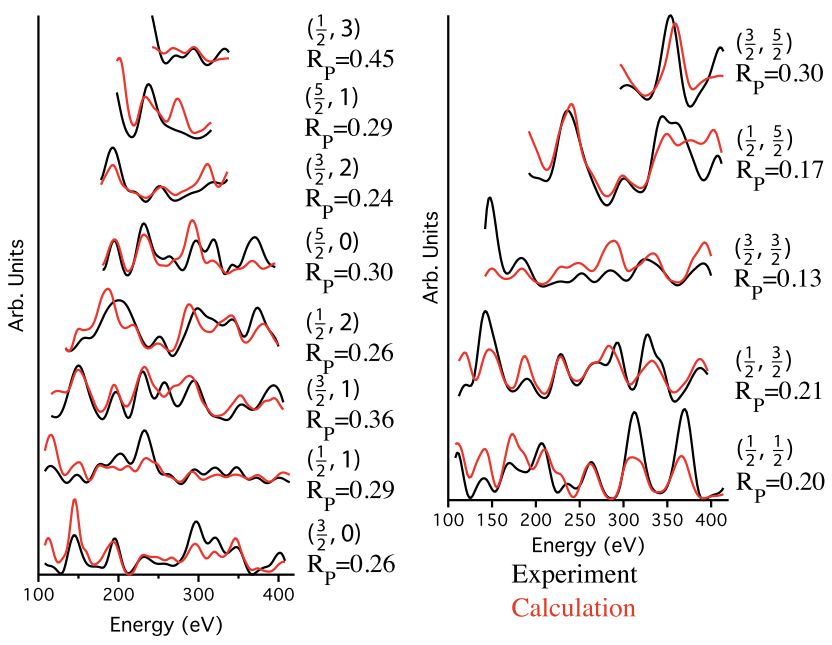

FIG. 2 (color online). Half integer IV curves for both terminations. Left-hand side: $2 \times 1$ termination; right-hand side: $\sqrt{2} \times \sqrt{2}$ termination. The Pendry $R$ factor is indicated for each spot.

atomic layers so as to give reliable estimates of the actual deviations from bulk positions proved to be feasible. We arrive at final Pendry $R$ factors of $R_{p}=0.19$ for the resulting distorted $\sqrt{2} \times \sqrt{2}$ termination and $R_{p}=0.29$ for the distorted $2 \times 1$ structure. IV simulation for a $2 \times 2$ reconstruction was unable to arrive at $R_{p}<0.5$ for any $2 \times 2$ structures we adopted. We also tried As terminated surfaces, also relaxing the atomic positions in the first three layers. This gives much higher Pendry $R$ factors, $R_{P} \approx 0.48$ for the $2 \times 1$ structure and $R_{P} \approx 0.42$ for the $\sqrt{2} \times \sqrt{2}$ structure. Therefore, based both on robust electrostatic considerations and our quantitative IV-LEED study, we can conclusively state that the termination surface of the Co-doped Ba122 superconductors cleaved at cryogenic temperatures consists of a half Ba layer coupled to relaxed atomic positions in the near-surface layers.

The atomic positions from IV-LEED are given in tables in the supplementary material [14], and clearly indicate significant deviations from the bulk structure. Apart from a small reduction in the interplanar distances, we also observe buckling of the outermost $\mathrm{As}-\mathrm{Fe}_{2}$-As block and lateral displacements of the atoms in the first four layers. The Debye temperature of the surface $\mathrm{Ba}$ atoms is found to be quite low $(60 \mathrm{~K})$, making these $\mathrm{Ba}$ atoms relatively mobile. This matches the observation that the fractional spots irreversibly fade out when the sample is warmed to approximately $150 \mathrm{~K}$ [10]. STM experiments also report destruction of the long-range order seen in the topographs by thermal cycling to high temperature [7]. Incidentally, this also explains why no clear fractional spots where observed in Ref. [9], as those samples were cleaved at room temperature. Seeing as almost all ARPES studies are conducted on cryogenically cleaved samples, the low $T$ structure reported here is of direct relevance.

Now that we have proven the existence of significant (sub)surface reconstructions, the next step is to quantify 
their effects on the electronic structure. This we do via DFT calculations for the different surface geometries using the full potential local orbital code (FPLO) [18] (for details see supplementary material [14]). We simulated the cleavage surface by using z-periodic replicas of a finite slab, separated by vacuum (i.e., repeated slab calculations). The structure of the slabs used in the calculations is shown in Fig. 3(a). In Figs. 3(a) and 3(b) we show only the top half of the slab: the other half is identical, but mirrored in the lowest $\mathrm{Ba}$ layer shown. To distinguish the effect of the Ba superstructure from the distortions, we repeated the calculations with similar slabs that have all atoms at the bulk positions, but with a $2 \times 1$ or $\sqrt{2} \times \sqrt{2}$ ordered halflayer of $\mathrm{Ba}$ at the surface. To address doping, the virtual crystal approximation is employed. The Ba superstructures impose a planar supercell on the whole slab, which has the consequence that all bands, including the bulk bands, are folded back into the smaller Brillouin zone of the supercell. Obviously, this backfolding becomes less and less meaningful as the layers become increasingly bulklike. In the following, we unfold the band structures according to the translational symmetry of the original planar unit cell using the approach of Ref. [19] (see supplementary material [14] for details). Figure 4(a) shows an experimental Fermi surface (FS) for the $x=0.17$ sample measured at $74 \mathrm{eV}$ photon energy. The DFT bulk FS is shown in Fig. 4(b). Comparing the two, we note two distinguishing features: (i) a weak FS sheet at the $X$ point in the experimental data and (ii) the fact that the electron FS around $M$ breaks the

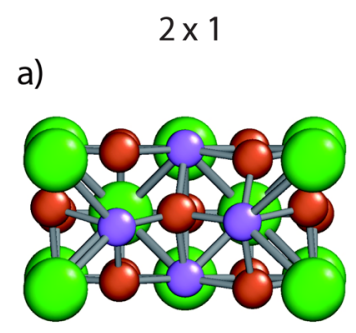

b)

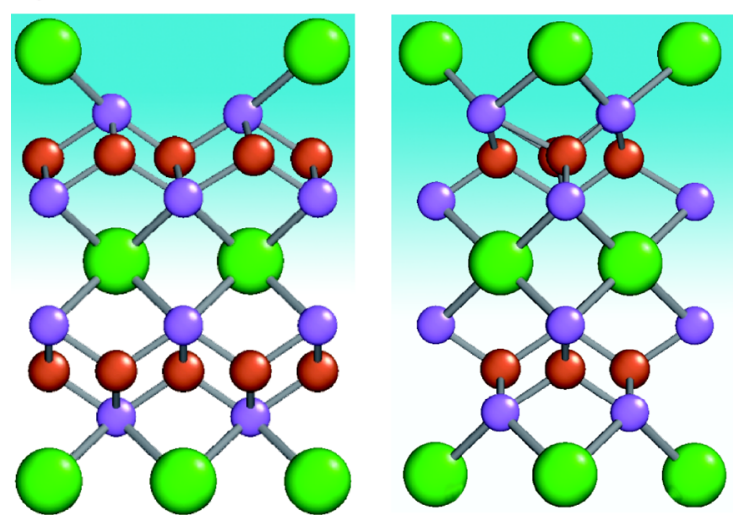

FIG. 3 (color online). Top view (a) and side view of half a slab (b) used in the DFT calculations (green, Ba; purple, As; orange, Fe). The blue shaded area in (b) indicates distorted atomic positions, as obtained from the LEED experiments (green, Ba; purple, As; orange, Fe. Coloring identical to Fig. 1). fourfold symmetry of the bulk crystal. The first feature has also been observed in $\mathrm{SrFe}_{2} \mathrm{As}_{2}$ [7], and disappeared when the sample was heated to $200 \mathrm{~K}$. The same feature is also in the Ca122 data of Ref. [20], making it a ubiquitous feature of the M122 iron pnictide family. Comparing with Fig. 4(c), we see that this feature arises from the $2 \times 1$ and $1 \times 2$ superstructures. At this stage, we cannot decide which out of the $2 \times 1$ or $\sqrt{2} \times \sqrt{2}$ structures is responsible for (ii), as both reconstructions lead to significant deviations from fourfold symmetry. We also note that we do not observe the large distortion-induced FS pocket encircling the $\Gamma$ point predicted by the calculations. More extensive variation of the experimental ARPES parameters (photon energy, polarization, geometry) will be necessary to judge to what extent this is a matrix element effect.

In Fig. 5, we compare an $I(k, E)$ image taken along the $\Gamma-M$ direction in 5(a) with the bands calculated for the 5(c) $2 \times 1$ and 5 (d) $\sqrt{2} \times \sqrt{2}$ distorted structures. As has been observed in all comparisons between ARPES and DFT calculations in the iron pnictides, one can identify most features if one includes a scaling factor renormalizing the energy scale of the DFT data by a factor of 2-3. Apart from this rescaling, there is good agreement between the calculated and measured spectra. In particular, we observe a surface state, indicated in Fig. 5(a) by the dashed yellow curve, that gives rise to the central peak in the displayed energy distribution curve. Further proof for the
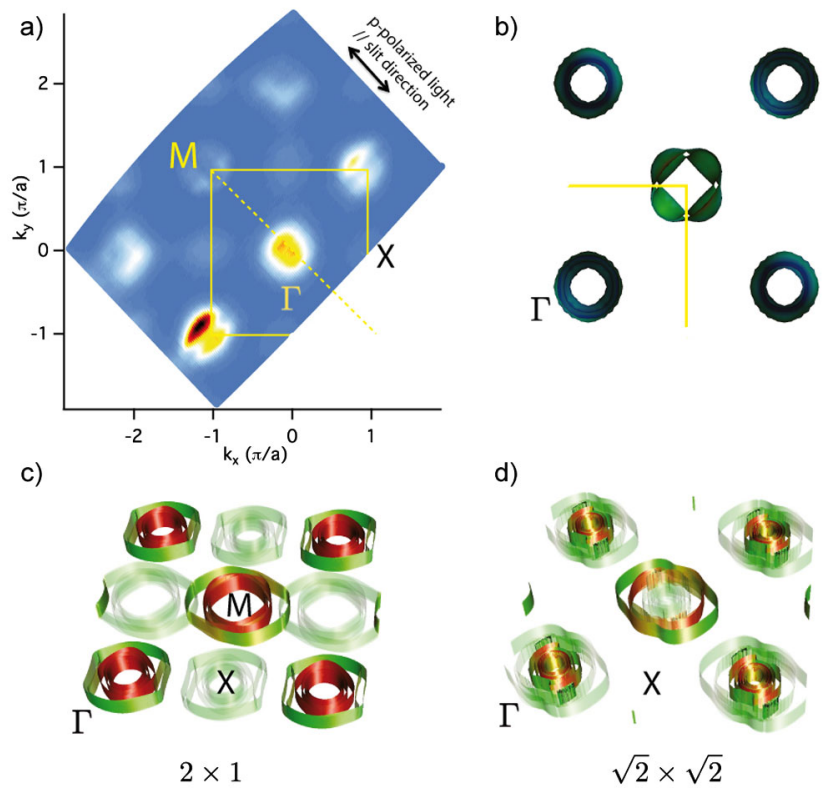

FIG. 4 (color online). (a) Fermi surface of $\mathrm{BaFe}_{1.83} \mathrm{Co}_{0.17} \mathrm{As}_{2}$ measured with $74 \mathrm{eV}, p$-polarized photons. Indicated in yellow is the 1st Brillouin zone and the $\Gamma$ and $M$ points. The dashed yellow line indicates the cuts shown in Fig. 5. (b) Undoped bulk FS from DFT. (c) Unfolded FS for the $2 \times 1$ termination, from DFT using the relaxed atomic positions and virtual crystal approximation doping of 0.17. Dark (red), bulk; light (green), surface; brown, mixture of bulk and surface states. (d) Same as (c), but for the $\sqrt{2} \times \sqrt{2}$ structure. 


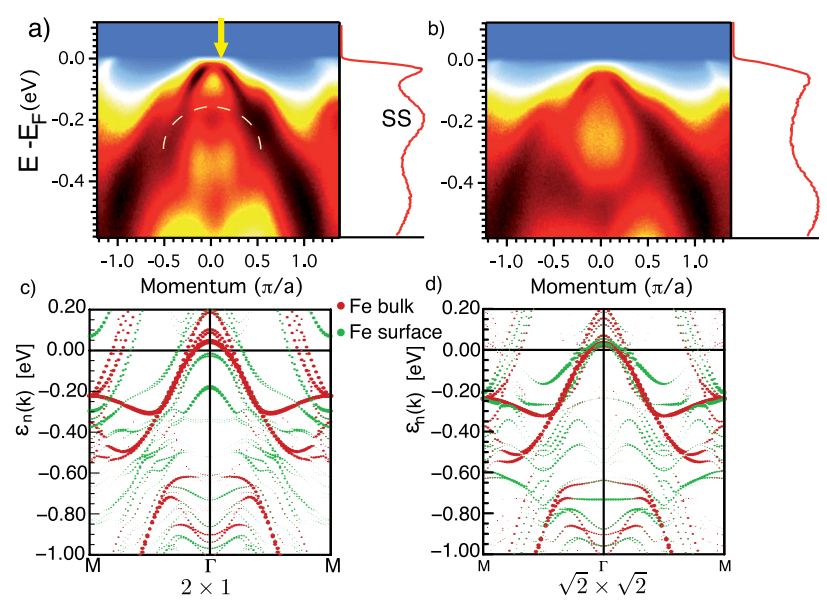

FIG. 5 (color online). (a) $I(k, E)$ image taken along the cut indicated in Fig. 4(a). On the side we show an energy distribution curve taken along the line indicated by the arrow. The dashed curve indicates the surface state (SS). (b) Same as (a) but taken after cycling in temperature. (c),(d) Calculated band structure for the same momentum window. Note that the energy window is twice as large for the DFT data.

identification of this band with surface-related states comes from Fig. 5(b), which is the same cut also measured at $10 \mathrm{~K}$, but taken after cycling the sample temperature to $150 \mathrm{~K}$. Like the fractional spots in the LEED experiments, the surface-related features fade away on the irreversible melting of the ordered $\mathrm{Ba}$ superstructure. Comparing the complexity of surface and bulk bands in 5(c) and 5(d) with the experimental data, several surface states probably contribute to the experimentally observed surface state. The closeness of the surface and bulk states will inevitably lead to a broadening of the ARPES spectra. It is likely that most of our conclusions carry over to other members of the 122 family, as both termination surfaces have been observed with STM. Ca122 appears to be somewhat special due to the fact that there is a larger energy separation between the $2 \times 1$ and $\sqrt{2} \times \sqrt{2}$ termination states [13]. This is also evident from STM experiments where only $2 \times 1$ termination is observed [21].

In conclusion, our combined IV-LEED (experiment and theory) and band mapping (experiment and theory) have clearly shown the surface of the Ba122-based high temperature pnictide superconductors to be not only comprised of a $1 / 2 \mathrm{Ba}$ layer, but also to be significantly distorted with respect to the bulk structure. This gives rise to surfacerelated bands, which can be clearly identified in ARPES data. These conclusions go a long way to resolving the riddle as to why so much of the published ARPES data on the Ba122 family is so broad: this is due to closely overlapping surface and bulk-derived states. This essentially excludes the use of the Ba122 system for accurate determination of, in particular, the imaginary part of the self-energies in these superconductors via ARPES.
We are grateful to $\mathrm{H}$. Luigjes for expert technical assistance. This work is part of the research program of the Foundation for Fundamental Research on Matter (FOM) and VENI program, which are part of the Netherlands Organisation for Scientific Research (NWO). The work in Dresden is carried out under the DFG priority program SPP1458.

*e.vanheumen@uva.nl.

[1] Y. Kamihara, T. Watanabe, M. Hirano, and H. Hosono, J. Am. Chem. Soc. 130, 3296 (2008).

[2] P. Tasker, J. Phys. C 12, 4977 (1979).

[3] A. Ohtomo and H. Huang, Nature (London) 427, 423 (2004).

[4] H. Eschrig, A. Lankau, and K. Koepernik, Phys. Rev. B 81, 155447 (2010)

[5] C. Liu, Y. Lee, A. D. Palczewski, J. Q. Yan, T. Kondo, B. N. Harmon, R. W. McCallum, T. A. Lograsso, and A. Kaminski, Phys. Rev. B 82, 075135 (2010).

[6] A. Lankau, K. Koepernik, S. Borisenko, V. Zabolotnyy, B. Büchner, J. van den Brink, and H. Eschrig, Phys. Rev. B 82, 184518 (2010).

[7] D. Hsieh, Y. Xia, L. Wray, D. Qian, K. Gomes, A. Yazdani, G. F. Chen, J.L. Luo, N.L. Wang, and M.Z. Hasan, arXiv:0812.2289v1.

[8] Y. Yin, M. Zech, T. L. Williams, X. F. Wang, G. Wu, X. H. Chen, and J.E. Hoffman, Phys. Rev. Lett. 102, 097002 (2009).

[9] V.B. Nascimento et al., Phys. Rev. Lett. 103, 076104 (2009).

[10] F. Massee, S. de Jong, Y. Huang, J. Kaas, E. van Heumen, J. B. Goedkoop, and M.S. Golden, Phys. Rev. B 80, 140507 (2009).

[11] H. Zhang et al., Phys. Rev. B 81, 104520 (2010).

[12] F. C. Niestemski, V.B. Nascimento, W. Plummer, J. Gillett, S. Sebastian, Z. Wang, and V. Madhavan, arXiv:0906.2761v1.

[13] M. Gao, F. Ma, Z.-Y. Lu, and T. Xiang, Phys. Rev. B 81, 193409 (2010).

[14] See supplemental material at http://link.aps.org/ supplemental/10.1103/PhysRevLett.106.027002 for details on experiments and a summary of the parameters of the calculations.

[15] Cleavage in the Fe plane is highly unlikely due to the much stronger bonding in the Fe-As block.

[16] A. Barbieri and M. van Hove, symmetrized automated tensor LEED package.

[17] J. Pendry, J. Phys. C 13, 937 (1980).

[18] K. Koepernik and H. Eschrig, Phys. Rev. B 59, 1743 (1999).

[19] W. Ku, T. Berlijn, and C.-C. Lee, Phys. Rev. Lett. 104, 216401 (2010).

[20] C. Liu et al., Phys. Rev. Lett. 102, 167004 (2009).

[21] T.-M. Chuang, M. P. Allan, J. Lee, Y. Xie, N. Ni, S. L. Bud'ko, G. S. Boebinger, P. C. Canfield, and J. C. Davis, Science 327, 181 (2010). 